\title{
Composición e invarianza factorial de la versión corta del Physical Self Description Questionnaire en adolescentes mexicanos
}

\section{Composition and factorial invariance of the Short Version of the Physical Self Description Questionnaire among Mexican adolescents}

\section{Estrutura e invariancia fatorial do versão curta de Physical Self Description Questionnaire em adolescentes mexicanos}

\author{
Ornelas, M.1, Benavides, E. V1, Solano-Pinto, N.2, Conchas, M.1, Rangel, Y. S.1, Blanco, H.1 \\ 1Facultad de Ciencias de la Cultura Física, Universidad Autónoma de Chihuahua, México; \\ 2Departamento Psicología Facultad de Educación, Campus Toledo, Universidad Castilla La Mancha, \\ España
}

\begin{abstract}
RESUMEN
El objetivo de esta investigación fue analizar las propiedades psicométricas propuestas por Marsh, Martin y Jackson (2010) para la versión corta del Physical Self Description Questionnaire (PSDQ-S). La muestra total fue de 915 adolescentes mexicanos 400 deportistas y 515 no deportistas, con edades comprendidas entre 11-15 años $(M=12.33$; $D E=1.43)$. La estructura factorial del cuestionario se analizó mediante análisis factoriales confirmatorios. Los análisis, muestran que una estructura de nueve factores (actividad física, apariencia, grasa corporal, coordinación, resistencia, flexibilidad, salud, competencia deportiva y fuerza) es viable y adecuada tanto para la muestra total (GFI .964; RMSEA .030; CFI .981) como para las poblaciones de adolescentes mexicanos deportistas (GFI .921; RMSEA .049; CFI .946) y no deportistas (GFI .943 y RMSEA .049; CFI .970). La estructura de nueve factores, atendiendo a criterios estadísticos y sustantivos, ha mostrado adecuados indicadores de ajuste de fiabilidad y validez. Por otro lado, la estructura factorial, las cargas factoriales y los interceptos se consideran invariantes de acuerdo a la variable práctica deportiva; sin embargo, existen diferencias significativas a favor de los deportistas en cuanto a su autoconcepto físico: actividad física $(-1.243, p<0.001)$, grasa corporal $(-0.212, p<0.05)$, coordinación $(-0.765, p$ $<0.001)$, resistencia $(-0.882, p<0.001)$, flexibilidad $(-0.427, p<0.001)$, salud $(-0.172, p<0.05)$, competencia deportiva $(-1.026, p<0.001)$ y fuerza $(-0.614, p<0.001)$. En conclusión, el PSDQ-S puede ser una herramienta útil para avanzar en el estudio de los factores que afectan al autoconcepto físico.
\end{abstract}

Palabras clave: validez; fiabilidad; psicometría; evaluación psicológica; autoconcepto físico.

Correspondence to: Humberto, Blanco, Vega. Dirección Postal: Paseos de Chihuahua, 31125 Chihuahua, Chih. Tel: +52 1614158 9900. Email: hblanco@uach.mx 


\title{
Ornelas, Benavides, Solano-Pinto, Conchas, Rangel, Blanco
}

\begin{abstract}
The aim of this research was to analyze the psychometric properties proposed by Marsh et al. (2010) for the Physical Self Description Questionnaire (PSDQ-S). The total sample has been composed by 915 Mexican adolescents, 400 athletes and 515 non-athletes, with ages from 11 to 15 years $(M=12.31, S D=1.43)$. The factor structure of questionnaire has been analized through the confirmatory factor analysis. This analysis shows that a nine factors structure is viable and adequate for the total sample (GFI .964; RMSEA .030; CFI .981) and the populations of athletes (GFI .921; RMSEA .049; CFI .946) and non-athletes (GFI .943 y RMSEA .049; CFI .970). The nine factors structure (physical activity, appearance, body fat, coordination, endurance, flexibility, health, sport competence, and strength), according to statistical and substantive criteria, has shown adequate indicators of reliability and validity adjustment. On the other hand, the factorial structure, the factorial loads and the intercepts are considered invariant according to the variable sports practice; however, there are differences between athletes and non-athletes in favor of athletes in terms of their physical self-concept: physical activity $(-1,243, p<0.001)$, body fat $(-0.212, \mathrm{p}<0.05)$, coordination $(-$ $0.765, p<0.001)$, endurance $(-0.882, \mathrm{p}<0.001)$, flexibility $(-0.427, p<0.001)$, health $(-0.172, \mathrm{p}<0.05)$, sports competition $(-1.026, p<0.001)$ and strength $(-0.614, p<0.001)$. In conclusion, the PSDQ-S can be a useful tool to advance in the study of the factors that affect the physical self-concept.
\end{abstract}

Keywords: validity; reliability; psychometics; psychological assessment; physical self-concept.

\section{RESUMO}

O principal objetivo deste estudo foi analizar as propiedades psicométricas propostas por Marsh et al. (2010) para a versão curta de Physical Self Description Questionnaire (PSDQ-S). Amostra total foi de 915 adolescentes mexicanos 400 atletas e 515 não- atletas com idades entre $11-15$ anos $(M=12.33 ; D E=1.43)$. A estrutura fatorial foi analisado com anàlise fatorial confirmatòria. Os analises mostran a estrutura do nove fatores (atividade física, aparência, gordura corporal, coordenação, resistencia, flexibidade, saúde, competencia esportiva e força) e factível e adequado para a mostra total (GFI .964; RMSEA .030; CFI .981) e para ambas as populações, atletas (GFI .921; RMSEA .049; CFI .946) e não atletas (GFI .943 y RMSEA .049; CFI .970). A estrutura de nove fatores, em resposta a criterios estatísticos e materiais tem mostrado indicadores de ajuste adequados de confiabilidade e validade. Além disso a estrutura de fatores, cargas fatoriais e intercepto são considerados invariantes em variável prativa esportiva, porém diferenças entre atletas e não atletas forom encontrados em favor dos atletas em termos de seu autoconceito físico: atividade física $(-1,243, p<0,001)$, gordura corporal $(-0,212, p<0,05)$, coordenação $(-0,765, p<0,001)$, resistência ($0,882, p<0,001)$, flexibilidade $(-0,427, p<0,001)$, saúde $(-0,172, p<0,05)$, competição esportiva $(-1,026, p<0,001)$ e força $(-0,614, p<0,001)$. Em conclusão, el PSDQ-S pode ser uma ferramenta útil para avançar no estudo dos fatores que afetam o autoconceito físico.

Palavras chave: validade; confiabilidade; psicometria; avaliação psicológica; autoconceito físico.

\section{INTRODUCCIÓN}

El autoconcepto físico (AFIS) se refiere a la forma en que una persona define su cuerpo en relación a aspectos funcionales del mismo y a su apariencia o atractivo físico (Murgui, García y García, 2016; Ries, 2011). El AFIS fue incorporado como una dimensión del autoconcepto general en las teorías de Shavelson, Hubner y Stanton (1976) teniendo una gran aceptación entre los distintos profesionales. Debido a dicha aceptación, proliferó la investigación respecto a la creación de instrumentos de medida como el Self
Description Questionnaires (SDQ, Marsh y Shavelson, 1985), cuestionario que evaluaba dos subescalas: Habilidad física y deportiva y apariencia física. Posteriormente se describió un modelo jerárquico y multifactorial basado en componentes específicos y generales que se convirtió en la base teórica para la nueva versión del SDQ denominado Physical Self- Concept Description Questionnaire (PSDQ, Marsh, Richards, Johnson, Roche y Tremayne, 1994) con el que se mide 9 componentes específicos (actividad física, apariencia, grasa 


\section{Composición e invarianza factorial de la versión corta del PSDQ-S}

corporal, coordinación, resistencia, flexibilidad, salud, competencia deportiva y fuerza), así como dos componentes globales: autoconcepto general y autoestima.

Los autores del cuestionario realizaron numerosos estudios para confirmar las propiedades psicométricas (Marsh, 1996) afirmando que es un cuestionario idóneo para ser utilizado en jóvenes a partir de 12 años, resaltando su invarianza factorial en grupos de hombres y mujeres y también entre atletas y no atletas (Marsh y Redmayne, 1994). Sin embargo, recomendaron la investigación en distintas muestras de diferentes procedencias para profundizar en las características psicométricas del instrumento (Marsh et al., 2010). De esta forma, investigaciones de distintos países han utilizado el PSDQ, como por ejemplo en muestras de los países de los Balcanes donde se concluye que los adolescentes son muy críticos respecto a su cuerpo y habilidades motoras (Janić et al., 2014) o en muestras procedentes de la India donde no encuentran relaciones entre composición corporal y AFIS en jóvenes adultos (Agarwal, Bhalla, Kaur y Babbar, 2013). Otras investigaciones han profundizado en el estudio psicométrico en muestras australianas y españolas, encontrando también invarianza factorial entre grupos de distintas culturas (Tomás, Marsh, González-Romá, Valls y Nagengast, 2014).

Puntuar alto en AFIS supone que la persona se percibe como capaz respecto a las habilidades de su cuerpo, es decir, que tiene fuerza, resistencia, competencia para hacer deporte y actividad física, con flexibilidad y coordinación para poder moverse con autonomía y seguridad, y en definitiva, que se percibe como una persona que está en forma y, además, con una apariencia física atractiva. De esta forma, los estudios han encontrado relación entre AFIS y conductas de autocuidado de la salud (Fernández-Bustos, GonzálezMartí, Contreras y Cueva, 2015; Peinado, Cocca, Solano-Pinto y Blanco, 2017). Otros estudios han considerado que el AFIS es una variable predictora respecto al estado general de salud (Trzesniewski et al., 2006; Urrutia, Azpillaga, de Cos y Muñoz, 2010) y en el uso saludable del tiempo libre (Biddle, Mutrie y Gorely, 2015; Fraguela-Vale, Varela-Garrote y Sanz-Arazuri, 2016) y la práctica de la actividad física (Aróstegi, Goñi, Infante y Zubillaga, 2013; Grao-
Cruces, Fernández-Martínez, Teva-Villén y Nuviala, 2017).

Uno de los ámbitos que más se ha estudiado ha sido la relación entre AFIS y el deporte y la actividad física, tanto en personas habitualmente deportistas, como no, intentando, la investigación, confirmar dicha relación y la dirección de la misma, es decir, si el AFIS produce un aumento en la práctica del ejercicio físico o si el deporte mejora el AFIS, aspecto que no se encuentra del todo concretado en la literatura científica, debido, entre otros, a la naturaleza descriptiva de los estudios en los que no se puede establecer relaciones de causaefecto entre las variables ((Reigal, Videra, Martín y Juárez, 2013). En esta línea, el Physical Self- Concept Description Questionnaire (PSDQ) es uno de los instrumentos de elección en programas de actividad física con la finalidad de comprobar los efectos del ejercicio físico sobre la mejora del AFIS en sus diferentes dimensiones. Por ejemplo, utilizando dicho instrumento y después de la participación de jóvenes en programas de actividad física y deporte, se han encontrado mejoras en las subdimensiones del AFIS en salud, apariencia física y fuerza (Mayorga-Vega, Viciana, Cocca y De Rueda, 2012), en apariencia física (Costigan, Eather, Plotnikoff, Hillman y Lubans, 2016), apariencia física, actividad y fuerza (McNamee, Timken, Coste, Tompkins y Peterson, 2016). Pero también existen estudios que no han encontrado ninguna mejora en AFIS utilizando como instrumento el PSDQ (Schneider, Dunton y Coope, 2008).

Por ello en la presente investigación instrumental (Ato, López y Benavente, 2013) se investiga la estructura factorial de la versión corta del Physical Self Description Questionnaire (PSDQ-S) propuesta por Marsh et al. (2010) y la equivalencia psicométrica del mismo en adolescentes mexicanos deportistas y no deportistas; lo que se justifica por la importancia de comprobar la estructura factorial de un instrumento y la equivalencia psicométrica del mismo en distintos grupos (Abalo, Lévy, Rial y Varela, 2006).

\section{MATERIAL Y MÉTODOS}

\section{Participantes}

La muestra de 915 adolescentes del norte de México, 400 de ellos practican algún deporte y participan regularmente en torneos o competencias deportivas. La muestra se obtuvo mediante un muestreo por 


\section{Ornelas, Benavides, Solano-Pinto, Conchas, Rangel, Blanco}

conveniencia. La edad de los participantes fluctuó entre los 11 y 15 años, con una media de 12.33 y una desviación estándar de 1.43 años.

\section{Instrumentos}

Versión corta del Physical Self Description Questionnaire (PSDQ-S) de Marsh et al. (2010), en su versión original, es un cuestionario de 40 ítems que evalúa el autoconcepto físico (AF), distribuidos en once subescalas: apariencia, grasa corporal, coordinación, resistencia, flexibilidad, salud, actividad física, competencia deportiva, fuerza, autoconcepto físico general y autoestima (las primeras nueve subescalas miden dimensiones específicas del AF, mientras que las dos últimas miden dimensiones generales del AF). El encuestado responde a cada uno de los 40 ítems, en una escala de 1 (falso) a 6 (verdadero), su grado de acuerdo con cada uno de los enunciados que se le proponen.

Para nuestro estudio, además de traducir los ítems del cuestionario, se hicieron dos adaptaciones a la versión de Marsh et al. (2010). Motivo por el cual nos referiremos al cuestionario PSDQ-S como cuestionario PSDQ-SM.

Primera adaptación, en la versión original las seis opciones de respuesta se puntúan 1 (Falso) a 6 (Verdadero); en la versión utilizada en la presente investigación las opciones de respuesta van de 0 (Totalmente Falso) a 5 (Totalmente Verdadero). Esta primera adaptación se justifica porque las personas asocian con mayor naturalidad al "0" con la categoría "Totalmente Falso".

La segunda adaptación consistió en aplicar el instrumento por medio de una computadora; esto con el fin de permitir el almacenamiento de los datos sin etapas previas de codificación, con una mayor precisión y rapidez.

\section{Procedimiento}

Una vez conseguido el permiso tanto de las autoridades educativas como el de las familias, se invitó a participar en el estudio a estudiantes de secundaria de la ciudad de La Paz Baja California Sur México. Los que aceptaron participar firmaron el consentimiento informado. Luego se aplicó el instrumento antes descrito en una sesión de aproximadamente 45 minutos; en las aulas de los centros educativos. Al inicio de la evaluación se comentaban las instrucciones del cuestionario y los objetivos de la investigación.

Una vez aplicado el instrumento se procedió a recopilar y a analizar los datos mediante el paquete SPSS 18.0 y AMOS 21.0.

\section{Análisis estadístico}

El análisis psicométrico se realizó en dos etapas: 1) Análisis de las propiedades psicométricas del instrumento y 2) Análisis de invarianza factorial; con el fin de obtener una prueba que presente las mejores propiedades psicométricas.

Análisis de las propiedades psicométricas del instrumento. El primer paso del análisis de las propiedades psicométricas del cuestionario consistió en calcular la asimetría y la curtosis de cada ítem, para determinar si se cumplía el supuesto de normalidad.

Luego, se sometieron a comparación dos modelos de medida: el PSDQ-SM11, que responde a una estructura de once factores acorde a la distribución original de los ítems dentro del cuestionario y el modelo PSDQ-SM9, que corresponde a la estructura factorial del modelo anterior sin los factores autoconcepto físico general y autoestima (por medir dimensiones generales y no específicas del AF), así como los ítems que de acuerdo a los índices de modificación resultaron no adecuados.

Posteriormente se calculó la fiabilidad de los factores del mejor de los modelos de medida obtenido, a través del Coeficiente Alpha de Cronbach (Elosua y Zumbo, 2008; Nunnally y Bernstein, 1995) y del Coeficiente Omega (Revelle y Zinbarg, 2009; Sijtsma, 2009). La validez convergente se estimó a través de la varianza media extraída (AVE).

Análisis de invarianza factorial. Con el fin de obtener una prueba que presente las mejores propiedades para la conformación de los puntajes del cuestionario PSDQ-SM en deportistas y no deportistas se llevó a cabo un análisis de la invarianza factorial, tomando como base el mejor modelo obtenido en la muestra total (modelo PSDQ-SM9). Luego se calculó la fiabilidad, en ambas muestras, de cada una de las dimensiones a través del Alfa de Cronbach y el 


\section{Composición e invarianza factorial de la versión corta del PSDQ-S}

Coeficiente Omega (Revelle y Zinbarg, 2009; Sijtsma, 2009).

Para conducir todos los análisis factoriales confirmatorios se utilizó el software AMOS 21 (Arbuckle, 2012), las varianzas de los términos de error fueron especificados como parámetros libres, en las variables latentes (factores) se fijó uno de los coeficientes estructurales asociados a uno, para que su escala sea igual a la de una de las variables observables (ítems). El método de estimación empleado fue el de Máxima Verosimilitud (ML) con la aplicación de procedimientos de remuestreo bootstrap para los casos de no normalidad (Byrne, 2016; Kline, 2011); aun cuando en AMOS 21.0 el ML es especialmente robusto para posibles casos de no normalidad, especialmente si la muestra es suficientemente amplia y los valores de asimetría y curtosis no son extremos (asimetría < $2 \mid$ y curtosis < |7|); siguiendo además la recomendación de (Thompson, 2004), en el sentido de que cuando se emplea análisis factorial confirmatorio se debe corroborar no sólo el ajuste de un modelo teórico sino que es recomendable comparar los índices de ajuste de varios modelos alternativos para seleccionar el mejor.

Para evaluar el ajuste del modelo se emplearon el estadístico Chi-cuadrado, el índice de bondad de ajuste $(G F I)$ y el error cuadrático medio de aproximación (RMSEA) como medidas absolutas de ajuste. El índice de bondad ajustado (AGFI), el Índice Tucker-Lewis $(T L I)$ y el índice de bondad de ajuste comparativo (CFI) como medidas de ajuste incremental. La razón de Chi-cuadrado sobre los grados de libertad $(C M I N / G L)$ y el Criterio de Información de Akaike (AIC) como medidas de ajuste de parsimonia (Byrne, 2016; Gelabert et al., 2011).

\section{RESULTADOS}

\section{Asimetría y curtosis de los ítems del cuestionario}

Todos los ítems muestran valores de asimetría de \pm $2.20 \mathrm{y} \pm 4.40$ de curtosis (Tabla 1 ), por lo que se infiere que se ajustan razonablemente a una distribución normal; por otro lado el índice multivariado de Mardia (432.86) por encima del valor 70 indica ausencia de normalidad multivariada (Rodríguez y Ruiz, 2008).

Tabla 1

Media, desviación estándar, asimetría y curtosis para los ítems del cuestionario.

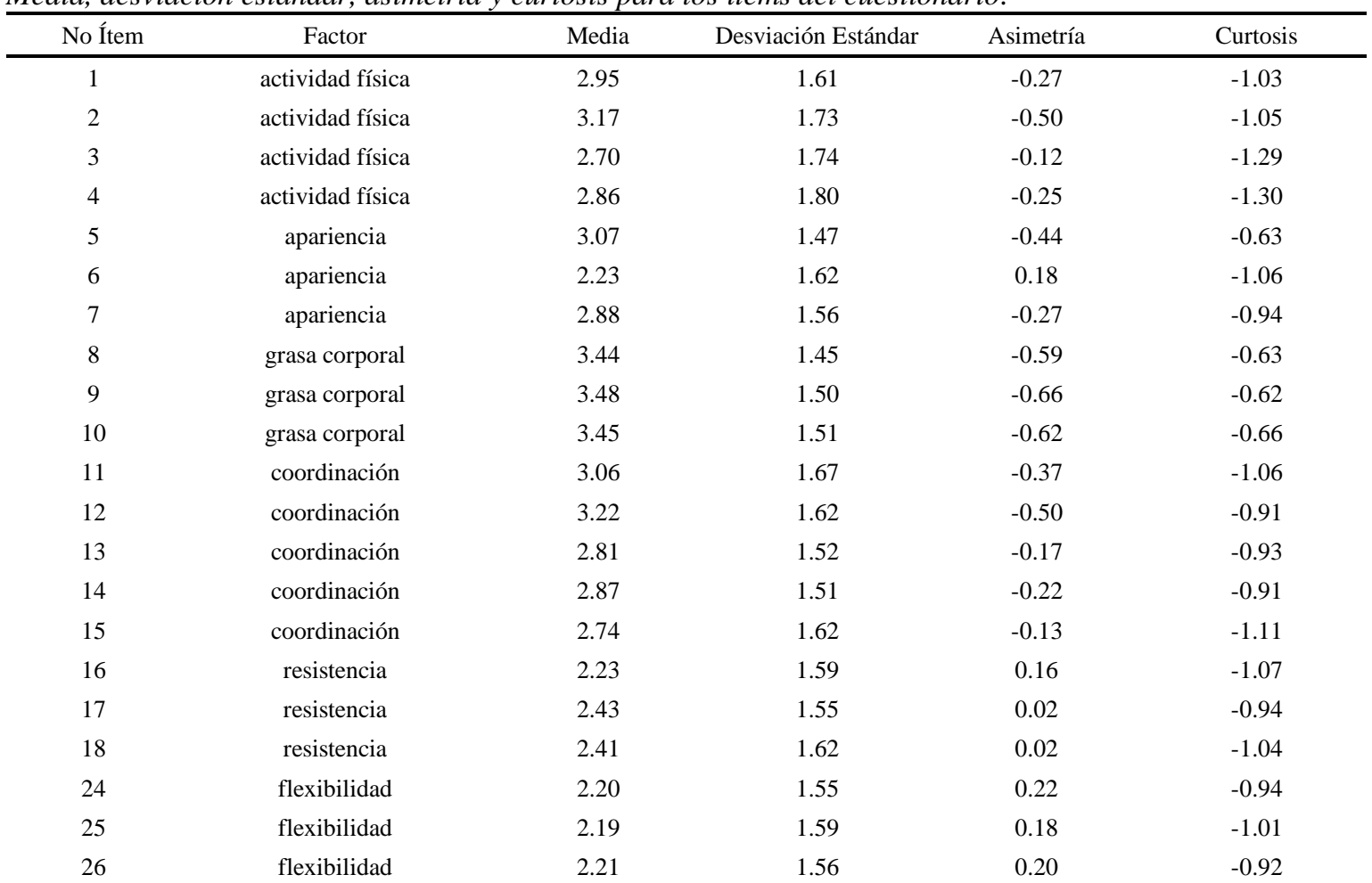




\section{Ornelas, Benavides, Solano-Pinto, Conchas, Rangel, Blanco}

Tabla 1

Media, desviación estándar, asimetría y curtosis para los ítems del cuestionario (continuación...).

\begin{tabular}{|c|c|c|c|c|c|}
\hline No Ítem & Factor & Media & Desviación Estándar & Asimetría & Curtosis \\
\hline 30 & salud & 3.96 & 1.30 & -1.35 & 1.33 \\
\hline 31 & salud & 4.28 & 1.28 & -1.99 & 3.31 \\
\hline 33 & salud & 3.72 & 1.40 & -1.08 & 0.47 \\
\hline 34 & salud & 4.02 & 1.37 & -1.45 & 1.33 \\
\hline 36 & competencia deportiva & 3.06 & 1.57 & -0.35 & -0.90 \\
\hline 37 & competencia deportiva & 2.84 & 1.57 & -0.23 & -1.00 \\
\hline 38 & fuerza & 2.54 & 1.46 & -0.05 & -0.79 \\
\hline 39 & fuerza & 2.67 & 1.45 & -0.14 & -0.71 \\
\hline 29 & autoconcepto físico general & 3.70 & 1.56 & -1.02 & -0.06 \\
\hline 19 & autoestima & 3.24 & 1.40 & -0.51 & -0.50 \\
\hline 20 & autoestima & 4.37 & 1.23 & -2.15 & 3.86 \\
\hline 21 & autoestima & 2.93 & 1.28 & -0.35 & -0.23 \\
\hline 22 & autoestima & 3.72 & 1.47 & -1.05 & 0.16 \\
\hline 23 & autoestima & 3.88 & 1.49 & -1.22 & 0.42 \\
\hline
\end{tabular}

\section{Análisis factorial confirmatorio muestra total}

Los resultados globales del análisis factorial confirmatorio (GFI .861; RMSEA .055; CFI .884) para el modelo PSDQ-SM11 que corresponde a una estructura de once factores acorde a la distribución original de los ítems dentro del cuestionario, indican que el modelo de medición no es aceptable (Tabla 2).

Tabla 2

Índices absolutos, incrementales y de parsimonia para los modelos generados.

\begin{tabular}{|c|c|c|c|c|c|c|c|c|}
\hline \multirow[b]{2}{*}{ Modelo } & \multicolumn{3}{|c|}{ Índices absolutos } & \multicolumn{3}{|c|}{ Índices incrementales } & \multicolumn{2}{|c|}{ Índices de parsimonia } \\
\hline & $\chi^{2}$ & GFI & RMSEA & AGFI & TLI & CFI & CMIN/DF & AIC \\
\hline $\begin{array}{l}\text { PSDQ- } \\
\text { SM11 }\end{array}$ & $2601.782 *$ & .861 & .055 & .842 & .870 & .884 & 3.754 & 2855.782 \\
\hline $\begin{array}{l}\text { PSDQ- } \\
\text { SM9 }\end{array}$ & $407.885 *$ & .964 & .030 & .952 & .977 & .981 & 1.829 & 561.885 \\
\hline
\end{tabular}

Nota: $* \mathrm{p}<.05 ; \mathrm{GFI}=$ índice de bondad de ajuste; RMSEA = error cuadrático medio de aproximación; AGFI = índice de bondad ajustado; TLI = índice de Tucker-Lewis; $\mathrm{CFI}$ = índice de ajuste comparativo; CMIN/DF = razón de Chi-cuadrado sobre los grados de libertad; AIC = criterio de información de Akaike

Los factores del modelo PSDQ-SM11 explican aproximadamente el $69 \%$ de la varianza y de acuerdo a los resultados obtenidos 17 de los 40 ítems saturan por debajo de .70 en su dimensión prevista (Tabla 3). 


\section{Composición e invarianza factorial de la versión corta del PSDQ-S}

Tabla 3

Soluciones estandarizadas análisis factorial confirmatorio para el Modelo PSDQ-SM11.

\begin{tabular}{|c|c|c|c|c|c|c|c|c|c|c|c|}
\hline Ítem & F1 & F2 & F3 & F4 & F5 & F6 & F7 & F8 & F9 & F10 & F11 \\
\hline \multicolumn{12}{|c|}{ Pesos Factoriales } \\
\hline 1. & .60 & & & & & & & & & & \\
\hline 2. & .70 & & & & & & & & & & \\
\hline 3. & .80 & & & & & & & & & & \\
\hline 4. & .79 & & & & & & & & & & \\
\hline 5. & & .66 & & & & & & & & & \\
\hline 6. & & .78 & & & & & & & & & \\
\hline 7. & & .86 & & & & & & & & & \\
\hline 8. & & & .78 & & & & & & & & \\
\hline 9. & & & .86 & & & & & & & & \\
\hline 10. & & & .86 & & & & & & & & \\
\hline 11. & & & & .56 & & & & & & & \\
\hline 12. & & & & .54 & & & & & & & \\
\hline 13. & & & & .78 & & & & & & & \\
\hline 14. & & & & .57 & & & & & & & \\
\hline 15. & & & & .74 & & & & & & & \\
\hline 16. & & & & & .73 & & & & & & \\
\hline 17. & & & & & .77 & & & & & & \\
\hline 18. & & & & & .74 & & & & & & \\
\hline 24. & & & & & & .76 & & & & & \\
\hline 25. & & & & & & .80 & & & & & \\
\hline 26. & & & & & & .79 & & & & & \\
\hline 30. & & & & & & & .58 & & & & \\
\hline 31. & & & & & & & .52 & & & & \\
\hline 32. & & & & & & & .68 & & & & \\
\hline 33. & & & & & & & .62 & & & & \\
\hline 34. & & & & & & & .68 & & & & \\
\hline 35. & & & & & & & & .78 & & & \\
\hline 36. & & & & & & & & .85 & & & \\
\hline 37. & & & & & & & & .77 & & & \\
\hline 38. & & & & & & & & & .71 & & \\
\hline 39. & & & & & & & & & .75 & & \\
\hline 40. & & & & & & & & & .77 & & \\
\hline 27. & & & & & & & & & & .69 & \\
\hline 28. & & & & & & & & & & .52 & \\
\hline 29. & & & & & & & & & & .59 & \\
\hline 19. & & & & & & & & & & & .71 \\
\hline 20. & & & & & & & & & & & .35 \\
\hline 21. & & & & & & & & & & & .59 \\
\hline 22. & & & & & & & & & & & .61 \\
\hline 23. & & & & & & & & & & & .30 \\
\hline
\end{tabular}

$$
\begin{aligned}
& \text { F1 - } \\
& \text { Correlaciones Factoriales } \\
& \text { F2 } \quad .38 \\
& \begin{array}{lll}
\mathrm{F} 3 & .07 \quad .11
\end{array} \\
& \begin{array}{lllll}
\mathrm{F} 4 & .73 & .53 & .14 & -
\end{array} \\
& \begin{array}{llll}
\text { F5 } & .76 & .47 & .21
\end{array} \quad .77
\end{aligned}
$$

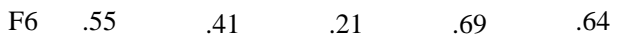

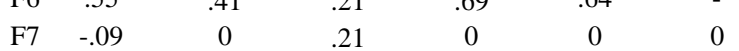

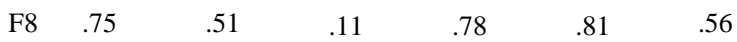

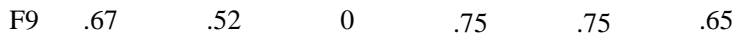

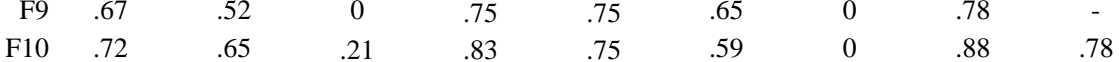

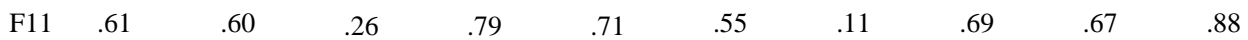




\section{Ornelas, Benavides, Solano-Pinto, Conchas, Rangel, Blanco}

Los resultados globales del análisis factorial confirmatorio (GFI .964; RMSEA .030; CFI .981) del segundo modelo sometido a prueba (PSDQ-SM9) que corresponde a la estructura factorial del modelo anterior, pero sin los factores autoconcepto físico general y autoestima sin los ítems $1,5,11,12,19$ al 23, 26 al 29, 31, 33 y 40 indican que este modelo de medición es mejor que el modelo anterior y que su ajuste es óptimo (Tabla 2). Los factores de este modelo explican aproximadamente el $75 \%$ de la varianza. Por otro lado solo cuatro de los ítems satura por debajo de .70 en su dimensión prevista (Tabla 4). Observándose además, en ambos modelos, intercorrelaciones de bajas a moderadas entre los factores evidenciando una adecuada validez discriminante entre ellos.

Tabla 4

Soluciones estandarizadas análisis factorial confirmatorio para el Modelo PSDQ-SM9.

\begin{tabular}{|c|c|c|c|c|c|c|c|c|c|}
\hline Ítem & F1 & $\mathrm{F} 2$ & $\mathrm{~F} 3$ & $\overline{\mathrm{F} 4}$ & F5 & F6 & F7 & F8 & F9 \\
\hline \multicolumn{10}{|c|}{ Pesos Factoriales } \\
\hline 2. & .70 & & & & & & & & \\
\hline 3. & .82 & & & & & & & & \\
\hline 4. & .81 & & & & & & & & \\
\hline 6. & & .82 & & & & & & & \\
\hline 7. & & .84 & & & & & & & \\
\hline 8. & & & .78 & & & & & & \\
\hline 9. & & & .86 & & & & & & \\
\hline 10. & & & .86 & & & & & & \\
\hline 13. & & & & .78 & & & & & \\
\hline 14. & & & & .55 & & & & & \\
\hline 15. & & & & .75 & & & & & \\
\hline 16. & & & & & .74 & & & & \\
\hline 17. & & & & & .78 & & & & \\
\hline 18. & & & & & .74 & & & & \\
\hline 24. & & & & & & .82 & & & \\
\hline 25. & & & & & & .80 & & & \\
\hline 30. & & & & & & & .62 & & \\
\hline 32. & & & & & & & .68 & & \\
\hline 34. & & & & & & & .65 & & \\
\hline 35. & & & & & & & & .78 & \\
\hline 36. & & & & & & & & .86 & \\
\hline 37. & & & & & & & & .76 & \\
\hline 38. & & & & & & & & & .78 \\
\hline 39. & & & & & & & & & .86 \\
\hline
\end{tabular}

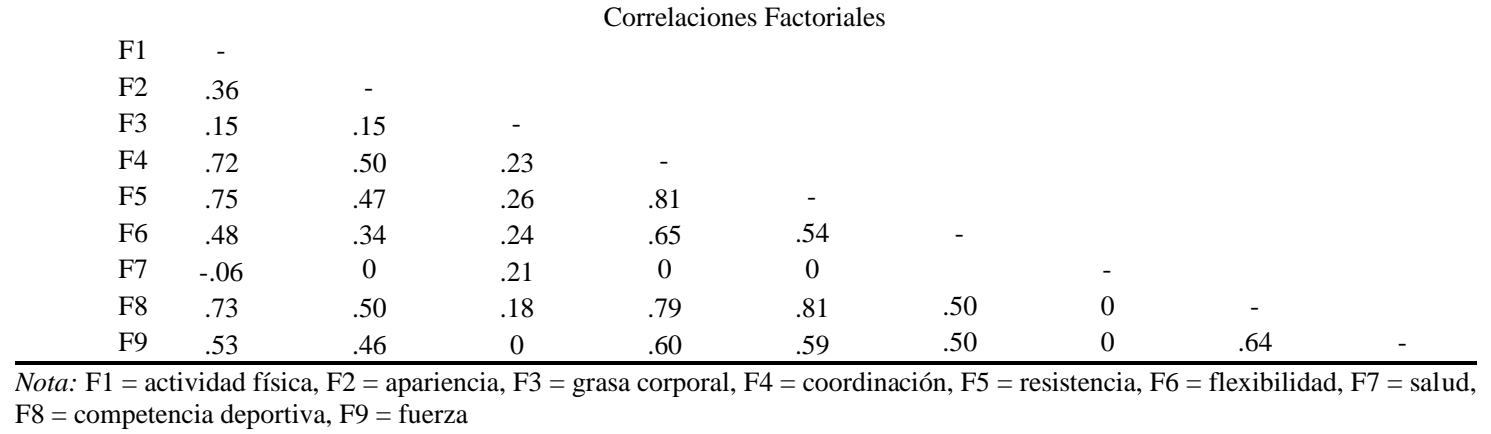

La mayoría de los factores obtenidos en los análisis factoriales confirmatorios, en ambos modelos, alcanzan valores de consistencia interna por encima de .75; evidenciando una consistencia interna adecuada para este tipo de subescalas (Tabla 5).

Tabla 5 


\section{Composición e invarianza factorial de la versión corta del PSDQ-S}

Coeficiente omega y alfa para los factores obtenidos en los Modelos PSDQ-SM11 y PSDQ-SM9.

\begin{tabular}{|c|c|c|c|c|}
\hline \multirow[b]{2}{*}{ Factor } & \multicolumn{2}{|c|}{ PSDQ-SM11 } & \multicolumn{2}{|c|}{ PSDQ-SM9 } \\
\hline & $\Omega$ & $\alpha$ & $\Omega$ & $\alpha$ \\
\hline Actividad física & .816 & .813 & .821 & .816 \\
\hline Apariencia & .813 & .802 & .816 & .817 \\
\hline Grasa corporal & .873 & .870 & .873 & .870 \\
\hline Coordinación & .777 & .772 & .739 & .730 \\
\hline Resistencia & .791 & .791 & .798 & .791 \\
\hline Flexibilidad & .827 & .825 & .792 & .786 \\
\hline Salud & .755 & .748 & .687 & .683 \\
\hline Competencia deportiva & .843 & .841 & .843 & .841 \\
\hline Fuerza & .788 & .789 & .805 & .798 \\
\hline Autoconcepto físico general & 630 & .651 & - & - \\
\hline Autoestima & 648 & .64 & - & - \\
\hline
\end{tabular}

\section{Análisis factoriales confirmatorios no deportistas y} deportistas

Tanto en la muestra de deportistas como de no deportistas todas las variables muestran valores de asimetría de \pm 2.00 y \pm 3.70 de curtosis, sin embargo, el índice multivariado de Mardia por encima del valor 70 indica ausencia normalidad multivariada (Rodríguez y Ruiz, 2008).

De acuerdo a los resultados de la Tabla 6 el análisis factorial confirmatorio de 24 ítems agrupados en nueve factores en la muestra de deportistas es aceptable (GFI .921 y RMSEA .049) y de acuerdo a las medidas de ajuste incremental y de parsimonia significativamente superior al modelo independiente y muy similar al modelo saturado.

Por otro lado, el análisis factorial confirmatorio en la muestra de no deportistas (Tabla 6), indica nuevamente que el modelo de medición de nueve factores es aceptable (GFI .943 y RMSEA .049) y de acuerdo a las medidas de ajuste incremental y de parsimonia significativamente superior al modelo independiente y muy similar al modelo saturado.

Tabla 6

Índices absolutos, incrementales y de parsimonia para los modelos generados. Análisis factorial confirmatorio para deportistas y no deportistas.

\begin{tabular}{|c|c|c|c|c|c|c|c|c|}
\hline \multirow[b]{2}{*}{ Modelo } & \multicolumn{3}{|c|}{ Índices absolutos } & \multicolumn{3}{|c|}{ Índices incrementales } & \multicolumn{2}{|c|}{ Índices de parsimonia } \\
\hline & $\chi^{2}$ & GFI & RMSEA & AGFI & TLI & CFI & CMIN/DF & AIC \\
\hline \multicolumn{9}{|c|}{ Solución factorial para los deportistas } \\
\hline 9 factores 24 ítems & $433.226^{*}$ & .921 & .049 & .894 & .933 & .946 & 1.943 & 587.226 \\
\hline Saturado & 0.000 & 1.000 & & & & 1.000 & & 600.00 \\
\hline Independiente & $4164.516^{*}$ & .345 & .188 & .288 & .000 & .000 & 15.089 & 4212.516 \\
\hline \multicolumn{9}{|c|}{ Solución factorial para los no deportistas } \\
\hline 9 factores 24 ítems & $379.836^{*}$ & .943 & .037 & .923 & .963 & .970 & 1.703 & 533.836 \\
\hline Saturado & 0.000 & 1.000 & & & & 1.000 & & 600.000 \\
\hline Independiente & $5516.510^{*}$ & .330 & .000 & .271 & .000 & .000 & 19.987 & 5564.510 \\
\hline
\end{tabular}

Nota: $* \mathrm{p}<.05 ; \mathrm{GFI}=$ índice de bondad de ajuste; RMSEA = error cuadrático medio de aproximación; AGFI = índice de bondad ajustado; TLI = índice de Tucker-Lewis; CFI = índice de ajuste comparativo; CMIN/DF = razón de Chi-cuadrado sobre los grados de libertad; $\mathrm{AIC}=$ criterio de información de Akaike 


\section{Ornelas, Benavides, Solano-Pinto, Conchas, Rangel, Blanco}

De acuerdo a los resultados de la Tabla 7, en ambas muestras, la mayoría de los ítems saturan por encima de .70 en su dimensión (factor) prevista.
Observándose además, intercorrelaciones moderadas entre los factores evidenciando una adecuada validez discriminante entre ellos.

Tabla 7

Soluciones estandarizadas para el análisis factorial confirmatorio en ambas muestras.

\begin{tabular}{|c|c|c|c|c|c|c|c|c|c|}
\hline Ítem & F1 & F2 & F3 & F4 & F5 & F6 & F7 & F8 & F9 \\
\hline & & & & Pesos F & toriales & & & & \\
\hline 2. & $.59 \mid .71$ & & & & & & & & \\
\hline 3. & $.82 \mid .79$ & & & & & & & & \\
\hline 4. & \begin{tabular}{l|l|}
.64 & .84
\end{tabular} & & & & & & & & \\
\hline 6. & & $.83 \mid .78$ & & & & & & & \\
\hline 7. & & $.88 \mid .86$ & & & & & & & \\
\hline 8. & & & $.78 \mid .78$ & & & & & & \\
\hline 9. & & & \begin{tabular}{l|l|}
.83 & .87
\end{tabular} & & & & & & \\
\hline 10. & & & $.90 \mid .83$ & & & & & & \\
\hline 13. & & & & $.74 \mid .79$ & & & & & \\
\hline 14. & & & & $.53 \mid .55$ & & & & & \\
\hline 15. & & & & $.71 \mid .74$ & & & & & \\
\hline 16. & & & & & \begin{tabular}{l|l}
.73 & .70
\end{tabular} & & & & \\
\hline 17. & & & & & $.71 \mid .78$ & & & & \\
\hline 18. & & & & & \begin{tabular}{l|l}
.66 & .77
\end{tabular} & & & & \\
\hline 24. & & & & & & $.79 \mid .81$ & & & \\
\hline 25. & & & & & & $.80 \mid .80$ & & & \\
\hline 30. & & & & & & & .67 | .60 & & \\
\hline 32. & & & & & & & 68 & & \\
\hline 34. & & & & & & & \begin{tabular}{l|l|}
.61 & .67
\end{tabular} & & \\
\hline 35. & & & & & & & & $.71 \mid .77$ & \\
\hline 36. & & & & & & & & $.83 \mid .86$ & \\
\hline 37. & & & & & & & & $.68 \mid .77$ & \\
\hline 38. & & & & & & & & & $.76 \mid .78$ \\
\hline 39. & & & & & & & & & $.87 \mid .83$ \\
\hline
\end{tabular}

Correlaciones Factoriales 


\section{Composición e invarianza factorial de la versión corta del PSDQ-S}

Invarianza de la estructura factorial entre deportistas y no deportistas

Los índices de ajuste obtenidos (Tabla 8) permiten aceptar la equivalencia de los modelos de medida básicos entre las dos muestras. Aunque el valor de Chi-cuadrado excede al exigido para aceptar la hipótesis de invarianza, los índices GFI=.933, $C F I=.960, R M S E A=.030$ y $A I C=1121.106$ contradicen esta conclusión lo que nos permite aceptar el modelo base de la invarianza (modelo sin restricciones).

Añadiendo al modelo base restricciones sobre las cargas factoriales caracterizamos la invarianza métrica. Los valores que se recogen en la Tabla 8 permiten aceptar este nivel de invarianza. El índice de ajuste general (GFI .931) y el error cuadrático medio de aproximación (RMSEA .030) siguen aportando información convergente en el sentido de la invarianza métrica. Además el criterio de información de Akaike (AIC 1123.463) y el índice comparativo de Bentler (CFI .958) no sufren grandes variaciones respecto al modelo anterior. Haciendo uso del criterio para la evaluación de los modelos anidados propuesto por (Cheung y Rensvold, 2002), quiénes sugieren que si el cálculo de la diferencia de los CFI de ambos modelos anidados disminuye en $.01 \mathrm{o}$ menos, se da por bueno el modelo restringido y por tanto el cumplimiento de la invarianza factorial; la diferencia entre CFIs obtenida permite aceptar el modelo de invarianza métrica. Podemos concluir hasta ahora que las cargas factoriales son equivalentes en las dos muestras.

Una vez demostrada la invarianza métrica entre las muestras, pasamos a evaluar la equivalencia entre interceptos (invarianza factorial fuerte). Los índices (Tabla 8) muestran un ajuste aceptable de este modelo, tanto evaluado de modo independiente como analizándolo respecto a su anidamiento con el modelo de invarianza métrica. La diferencia entre los índices comparativos de Bentler es de dos milésimas; el índice de ajuste general es .924 y el error cuadrático medio de aproximación es .031. Aceptada la invarianza fuerte, los dos modelos evaluados son equivalentes respecto a los coeficientes factoriales y a los interceptos.
Tabla 8

Índices de bondad de ajuste de cada uno de los modelos puestos a prueba en la invarianza factorial.

\begin{tabular}{|c|c|c|c|c|c|c|c|}
\hline \multirow[t]{2}{*}{ Modelo } & \multicolumn{7}{|c|}{ Índice de Ajuste } \\
\hline & $\chi^{2}$ & gl & GFI & NFI & CFI & RMSEA & AIC \\
\hline $\begin{array}{l}\text { Modelo sin } \\
\text { restricciones }\end{array}$ & $813.106^{*}$ & 446 & .933 & .916 & .960 & .030 & 1121.106 \\
\hline Invarianza métrica & $845.463 *$ & 461 & .931 & .913 & .958 & .030 & 1123.463 \\
\hline $\begin{array}{l}\text { Invarianza } \\
\text { factorial fuerte }\end{array}$ & $940.744 *$ & 499 & .924 & .903 & .952 & .031 & 1142.744 \\
\hline
\end{tabular}

Los factores obtenidos en los análisis factoriales confirmatorios alcanzan valores de consistencia interna por encima de .75 en ambas muestras (deportistas y no deportistas), en cuanto a la varianza media extraída la mayoría de los factores obtienen valores por encima de .50 (Tabla 9).

Tabla 9

Coeficiente omega y alfa para el factor obtenido.

\begin{tabular}{lcccccc}
\hline \multirow{2}{*}{ Factor } & \multicolumn{3}{c}{ Deportistas } & \multicolumn{3}{c}{ No Deportistas } \\
\cline { 2 - 8 } Actividad física & $\Omega(\mathrm{FC})$ & $\alpha$ & AVE & $\Omega(\mathrm{FC})$ & $\alpha$ & AVE \\
\cline { 2 - 7 } Apariencia & .735 & .727 & .485 & .824 & .820 & .611 \\
Grasa corporal & .845 & .842 & .732 & .805 & .796 & .674 \\
Coordinación & .876 & .875 & .702 & .867 & .866 & .685 \\
Resistencia & .702 & .695 & .444 & .739 & .723 & .491 \\
Flexibilidad & .743 & .739 & .491 & .795 & .787 & .564 \\
Salud & .775 & .776 & .632 & .786 & .785 & .648 \\
Competencia & .687 & .680 & .423 & .687 & .682 & .424 \\
deportiva & .786 & .772 & .552 & .843 & .836 & .642 \\
Fuerza & .800 & .793 & .667 & .787 & .782 & .649 \\
\hline
\end{tabular}

Nota $:$ FC $=$ fiabilidad compuesta AVE = varianza media extraída

Contrastes de las medias de los factores entre deportistas y no deportistas

Una vez comprobada la invarianza factorial, las diferencias entre las medias del factor de los dos grupos se estimaron tomando como referente la muestra de deportistas, fijando en 0 el valor de las medias para dicha muestra y estimando libremente el valor de las medias para la muestra de no deportistas. Las restricciones sobre los coeficientes de regresión e 


\section{Ornelas, Benavides, Solano-Pinto, Conchas, Rangel, Blanco}

interceptos, requeridos para los contrastes entre las medias se realizaron automáticamente mediante el software AMOS 21 (Arbuckle, 2012). Los resultados de las comparaciones indicaron que las medias de los factores actividad física $(-1.243, p<0.001)$, grasa corporal $(-0.212, p<0.05)$, coordinación $(-0.765$, $p<0.001)$, resistencia $(-0.882, p<0.001)$, flexibilidad ($0.427, p<0.001)$, salud $(-0.172, p<0.05)$, competencia deportiva $(-1.026, p<0.001)$ y fuerza $(-0.614, p<0.001)$ fueron significativamente mayores en los deportistas y sin diferencias en el factor apariencia $(-.173, p>0.05)$.

\section{DISCUSIÓN Y CONCLUSIONES}

De los resultados mostrados, de su análisis y tomando en cuenta que el objetivo principal de este estudio fue el de examinar la estructura factorial de la versión corta del Physical Self Description Questionnaire (PSDQ-SM) y la medición de su invariancia factorial en adolescentes mexicanos deportistas y no deportistas, se pueden obtener las siguientes conclusiones:

1) Los análisis factoriales confirmatorios realizados en la muestra total apoyan la estructura factorial de nueve factores (actividad física, apariencia, grasa corporal, coordinación, resistencia, flexibilidad, salud, competencia deportiva y fuerza); donde los factores así obtenidos presenta saturaciones factoriales estandarizadas adecuadas, saturaciones que, en general, se corresponden con la estructura propuesta para el cuestionario por Marsh et al. (2010) a pesar de eliminar dos factores y dieciséis de los ítems. Además, los factores correlacionan entre sí de forma positiva y estadísticamente significativa lo cual muestra que a medida que mejora la percepción del autoconcepto físico en alguna de las dimensiones, también mejora en las otras.

2) El análisis factorial confirmatorio tanto en la muestra de deportistas como la de no deportistas, indicó que el ajuste de los datos al modelo teórico de 24 ítems agrupados en nueve factores es aceptable, así como los factores obtenidos presentan saturaciones factoriales estandarizadas adecuadas. Estos resultados indican que el instrumento es adecuado para evaluar, tanto muestras de deportistas, como no deportistas, tal y como ya indicaban algunos estudios (Marsh y Redmayne, 1994; Tomás et al., 2014).
3) Los factores, en ambas muestras, evidenciaron una consistencia interna aceptable, a pesar del número reducido de ítems en cada uno de ellos.

4) Conjuntamente con todo lo antes dicho, los resultados del análisis de la invarianza factorial entre deportistas y no deportistas; indican una alta congruencia entre pares de factores. Lo que sugiere la existencia de fuertes evidencias de la validación cruzada de la medida y por tanto de la estabilidad de la estructura.

5) Las comparaciones entre los grupos reflejaron diferencias significativas a favor de los deportistas en cuanto a su autoconcepto físico. Este resultado apunta a los posibles beneficios que diferentes investigaciones han señalado sobre la práctica de actividad físico-deportiva y la salud de los adolescentes, en concreto, el autoconcepto físico (Biddle et al., 2015; Fernández-Bustos et al., 2015; Grao-Cruces et al., 2017; Trzesniewski et al., 2006). Los resultados obtenidos apoyan a los estudios que valoran la práctica de la actividad físico-deportiva como un factor positivo para la salud, potenciando los efectos positivos sobre el autoconcepto físico (Costigan et al., 2016; Mayorga-Vega et al., 2012; McNamee et al., 2016). El cuestionario PSDQ- SM puede ser un instrumento adecuado para determinar qué características deben tener los programas para promocionar el deporte y la actividad física y qué dimensiones del AFIS mejoran a través de dichos programas (Reigal et al., 2013).

En síntesis, el análisis de las propiedades psicométricas del cuestionario PSDQ-SM ha mostrado que una estructura de nueve factores, de acuerdo a los requisitos psicométricos establecidos, es viable, adecuada e invariante entre adolescentes deportistas y no deportistas. La estructura de nueve factores ha mostrado adecuados indicadores de ajuste $y$ de validez.

Sin embargo, cuando menos dos limitaciones están presentes en este trabajo. La primera es que los participantes son solo estudiantes, lo que supone una amenaza para la posibilidad de generalizar estos resultados. Ampliar la muestra (agregando por ejemplo adolescentes que no sean estudiantes) es un área de trabajo de cara al futuro. La segunda limitación proviene del propio instrumento de medición, que se 


\section{Composición e invarianza factorial de la versión corta del PSDQ-S}

basa en el autoinforme y que por ello puede contener los sesgos que se derivan de la deseabilidad social.

Asimismo, es indispensable comprobar si el AFIS, evaluado mediante el cuestionario PSDQ- SM, puede predecir conductas saludables.

\section{APLICACIONES PRÁCTICAS}

Mejorar el autoconcepto físico de los adolescentes, sin duda, contribuye a su calidad de vida, de ahí la necesidad de contar con instrumentos válidos y confiables para su medición. Por ello, el presente estudio analiza las propiedades psicométricas propuestos por Marsh et al. (2010) para la versión corta del Physical Self Description Questionnaire (PSDQ-SM). Este estudio además sirve de premisa para futuras investigaciones sobre el estudio de instrumentos para la medición del autoconcepto físico en poblaciones con diferentes factores personales y culturales. Finalmente, el presente instrumento será de gran utilidad para la aplicación en diferentes ámbitos como, por ejemplo, estudios descriptivos o de intervención.

\section{AGRADECIMIENTOS}

La Secretaría de Educación Pública-Subsecretaría de Educación Superior-Dirección de Superación Académica- Programa para el Desarrollo Profesional Docente (DE-13 -6894) financió este estudio. Así como la facilidad para la financiación de la publicación (CONACYT, Redes Temáticas, No. 269614).

\section{REFERENCIAS}

1. Abalo, J., Lévy, J., Rial, A. y Varela, J. (2006). Invarianza factorial con muestras múltiples. En J. Lévy (Ed.), Modelización con Estructuras de Covarianzas en Ciencias Sociales (pp. 259-278). Madrid: Netbiblo.

2. Agarwal, S., Bhalla, P., Kaur, S. y Babbar, R. (2013). Effect of body mass index on physical self concept, cognition \& academic performance of first year medical students. Indian Journal of Medical Research, 138(4), 515-522.

3. Arbuckle, J. R. (2012). AMOS users guide version 21.0. Chicago, IL: Marketing Department, SPSS Incorporated.
4. Aróstegi, B., Goñi, A., Infante, G. y Zubillaga, A. (2013). El autoconcepto físico de jóvenes futbolistas de alto rendimiento. Cuadernos de Psicología del Deporte, 13(1), 9-13.

5. Ato, M., López, J. J. y Benavente, A. (2013). Un sistema de clasificación de los diseños de investigación en psicología. Anales de Psicología, 29(3), 1038-1059.

6. Biddle, S. J. H., Mutrie, N. y Gorely, T. (2015). Psychology of Physical Activity Determinants, well-being and interventions. Oxford: Routledge.

7. Byrne, B. M. (2016). Structural Equation Modeling With AMOS: Basic Concepts, Applications, and Programming. New York, NY: Routledge.

8. Costigan, S. A., Eather, N., Plotnikoff, R. C., Hillman, C. H. y Lubans, D. R. (2016). HighIntensity Interval Training for Cognitive and Mental Health in Adolescents. Medicine \& Science in Sports \& Exercise, 48(10), 19851993. doi: 10.1249/MSS.0000000000000993

9. Cheung, G. W. y Rensvold, R. B. (2002). Evaluating goodness-of-fit indexes for testing measurement invariance. Structural Equation Modeling, 9(2), 233-255. doi: 10.1207/s15328007SEM0902_5

10. Elosua, P. y Zumbo, B. D. (2008). Coeficientes de fiabilidad para escalas de respuesta categórica ordenadas. Psicothema, 20(4), 896-901.

11. Fernández-Bustos, J.-G., González-Martí, I., Contreras, O. y Cueva, R. (2015). Relación entre imagen corporal y autoconcepto físico en mujeres adolescentes. Revista Latinoamericana de Psicología, 47(1), 25-33.

12. Fraguela-Vale, R., Varela-Garrote, L. y SanzArazuri, E. (2016). Ocio deportivo, imagen corporal y satisfacción vital en jóvenes españoles. Revista de Psicología del Deporte, 25(2), 33-38.

13. Gelabert, E., García-Esteve, L., MartínSantos, R., Gutiérrez, F., Torres, A. y Subirà, S. (2011). Psychometric properties of the Spanish version of the Frost Multidimensional 


\section{Ornelas, Benavides, Solano-Pinto, Conchas, Rangel, Blanco}

Perfectionism Scale in women. Psicothema, 23(1), 133-139.

14. Grao-Cruces, A., Fernández-Martínez, A., Teva-Villén, M. R. y Nuviala, A. (2017). Autoconcepto físico e intencionalidad para ser físicamente activo en los participantes del programa escuelas deportivas. Journal of sport and health research, 9(1), 15-26.

15. Janić, S. R., Jurak, G., Milanovic, I., Lazarevic, D., Kovač, M. y Novak, D. (2014). Physical self-concept of adolescents in Western Balkan countries: a pilot study. Perceptual and Motor Skills, 119(2), 629-649. doi: 10.2466/08.PMS.119c23z7

16. Kline, R. B. (2011). Principles and practice of structural equation modeling. New York: Guilford Press.

17. Marsh, H. W. (1996). Construct Validity of Physical Self-Description Questionnaire Responses: Relations to External Criteria. Journal of Sport \& Exercise Psychology, 18(2), 111-131.

18. Marsh, H. W., Martin, A. J. y Jackson, S. (2010). Introducing a Short Version of the Physical Self Description Questionnaire: new strategies, short-form evaluative criteria, and applications of factor analyses. Journal of Sport \& Exercise Psychology, 32(4), 438-482.

19. Marsh, H. W. y Redmayne, R. S. (1994). A Multidimensional Physical Self-concept and Its Relations to Multiple Components of Physical Fitness. Journal of Sport \& Exercise Psychology, 16(1), 45-55.

20. Marsh, H. W., Richards, G. E., Johnson, S., Roche, L. y Tremayne, P. (1994). Physical Self-Description Questionnaire: psychometric properties and a multitrait-multimethod analysis of relation to existing instruments. Journal of Sport \& Exercise Psychology, 16(3), 270-305.

21. Marsh, H. W. y Shavelson, R. J. (1985). Selfconcept: Its multifaceted, hierarchical structure. Educational Psychologist, 20(3), 107-123.

22. Mayorga-Vega, D., Viciana, J., Cocca, A. y De Rueda, B. (2012). Effect of a Physical
Fitness Program on Physical Self-Concept and Physical Fitness Elements in Primary School Students. Perceptual and Motor Skills, 115(3), 984-996. doi: 10.2466/06.10.25.PMS.115.6.984-996

23. McNamee, J., Timken, G. L., Coste, S. C., Tompkins, T. L. y Peterson, J. (2016). Adolescent girls' physical activity, fitness and psychological well-being during a health club physical education approach. European Physical Education Review, 23(4), 517-533. doi: $10.1177 / 1356336 X 16658882$

24. Murgui, S., García, C. y García, Á. (2016). Efecto de la práctica deportiva en la relación entre las habilidades motoras, el autoconcepto físico y el autoconcepto multidimensional. Revista de Psicología del Deporte, 25(1), 1925.

25. Nunnally, J. C. y Bernstein, I. H. (1995). Teoría Psicométrica. México: McGraw-Hill.

26. Peinado, J. E., Cocca, A., Solano-Pinto, N. y Blanco, H. (2017). Invarianza factorial de una escala de autoeficacia en deportistas y no deportistas. Revista de Psicología del Deporte, 26(2), 189-187.

27. Reigal, R., Videra, A., Martín, I. y Juárez, R. (2013). Importancia del autoconcepto físico y la autoeficacia general en la predicción de la conducta de práctica física. Apunts. Educación Física y Deportes, 112(2), 46-51. doi: $\quad 10.5672 /$ apunts.20140983.es.(2013/2).112.03

28. Revelle, W. y Zinbarg, R. E. (2009). Coefficients alpha, beta, omega and the glb: comments on Sijtsma. Psychometrika, 74(1), 145-154. doi: 10.1007/s11336-008-9102-z

29. Ries, F. (2011). El autoconcepto físico en adolescentes sevillanos en función del sexo y de la evolución de la carrera deportivo. Retos(19), 38-42.

30. Rodríguez, M. N. y Ruiz, M. A. (2008). Atenuación de la asimetría y de la curtosis de las puntuaciones observadas mediante transformaciones de variables: Incidencia sobre la estructura factorial. Psicológica, 29, 205-227. 


\section{Composición e invarianza factorial de la versión corta del PSDQ-S}

31. Schneider, M., Dunton, G. F. y Coope, D. M. (2008). Physical Activity and Physical SelfConcept among Sedentary Adolescent Females; An Intervention Study. Psychology of Sport and Exercise, 9(1), 1-14. doi: 10.1016/j.psychsport.2007.01.003

32. Shavelson, R. J., Hubner, J. J. y Stanton, G. C. (1976). Self concept: Validation of construct interpretations. Review of Educational Research, 46, 407-441.

33. Sijtsma, K. (2009). On the use, the misuse, and the very limited usefulness of Cronbach's alpha. Psychometrika, 74(1), 107-120. doi: 10.1007/s11336-008-9101-0

34. Thompson, B. (2004). Exploratory and Confirmatory Factor Analysis. Understanding concepts and applications. . Washington, D C: American Psychological Association.

35. Tomás, I., Marsh, H. W., González-Romá, V., Valls, V. y Nagengast, B. (2014). Testing Measurement Invariance Across Spanish and English Versions of the Physical SelfDescription Questionnaire: An Application of Exploratory Structural Equation Modeling. Journal of Sport \& Exercise Psychology, 36(2), 179-188. doi: 10.1123/jsep.2013-0070

36. Trzesniewski, K. H., Donnellan, M. B., Moffitt, T. E., Robins, R. W., Poulton, R. y Caspi, A. (2006). Low self-esteem during adolescence predicts poor health, criminal behavior, and limited economic prospects during adulthood. Developmental Psychology, 42(2), 381-390. doi: 10.1037/0012-1649.42.2.381

37. Urrutia, S., Azpillaga, I., de Cos, G. L. y Muñoz, D. (2010). Relación entre la percepción de estado de salud con la práctica físicodeportiva y la imagen corporal en adolescentes. Cuadernos de Psicología del Deporte, 20 (2), 51-56. 